\title{
Making Use of Abstract \\ Concepts-Systemic-Functional Linguistics and Ambient Intelligence
}

\author{
Jörg Cassens and Rebekah Wegener
}

\begin{abstract}
One of the challenges for ambient intelligence is to embed technical artefacts into human work processes in such a way that they support the sense making processes of human actors instead of placing new burdens upon them. This successful integration requires an operational model of context. Such a model of context is particularly important for disambiguating abstract concepts that have no clear grounding in the material setting of the work process. This paper examines some of the strengths and current limitations in a systemic functional model of context and concludes by suggesting that the notions of instantiation and stratification can be usefully employed.
\end{abstract}

\section{Introduction}

The exhibition of intelligent seeming behaviour is necessary for an artefact to be considered intelligent. Intelligent seeming behaviour is generally considered to be behaviour that is contextually appropriate. An ability to accurately read context is important for any animal if it is to survive, but it is especially important to social animals and of these perhaps humans have made the most out of being able to read context, where such an ability is tightly linked to reasoning and cognition [1].

The necessity of exhibiting some kind of intelligent behaviour has lead to the developments jointly labelled as ambient intelligence [2]. But to successfully create intelligent artefacts, the socio-technical processes and their changes through the use of mediating artefacts have to be examined more closely. This paper focuses on

\footnotetext{
Jörg Cassens

Department of Computer and Information Science, Norwegian University of Science and Technology, 7491 Trondheim, Norway e-mail: cassens@idi.ntnu.no

Rebekah Wegener

Centre of Language in Social Life, Department of Linguistics, Macquarie University NSW 2109, Australia e-mail: rwegener@ling.mq.edu.au
} 
how a social-semiotic theory of language, in which context is seen as integral to understanding communication, can be usefully employed in ambient intelligence. Ambient intelligence and its requirements from semiotics is further discussed in section 2 below.

Semiotics, or the study of sign systems, is here examined using a systemic functional model (see section 3). Systemic functional linguistics is a social semiotic theory of language which treats all behaviour as meaning bearing. This includes the behaviour of non-human participants and is oriented to the shared rather than the unique aspects of sign systems. The relationship between semiotics and ambient intelligence is outlined in section 4 below. In this paper we discuss how a systemic functional approach to semiotics is valuable in defining abstract concepts, see section 5. Abstract concepts, or concepts which have no direct referent in the material setting, are an important part of the mental tool set for humans. They allow us to transcend the here and now by providing us with a shorthand for complex events or complex sets of ideas. Despite this benefit, they do represent a challenge for modelling within ambient intelligence. Because they lack a clear material referent, abstract concepts are difficult to disambiguate and respond appropriately to. We propose that a systemic functional model of context will be beneficial in understanding abstract concepts.

We conclude this paper by pointing to future work in this area. For example, while we have focused on devices designed to interact closely with a single user, humans typically interact in groups, so it will be necessary to consider the impact of this for environments where not all users share the same meaning system.

\section{Ambient Intelligence}

In understanding human cognition and reasoning, disciplines such as neuroscience, psychology, sociology, linguistics, and philosophy have had to take a stance on context as a concept. Setting aside the more mechanistic views taken on reasoning, which typically need not consider context at all, positions on context tend to fall into two broad domains: those who see context as vast and unable to be coded and those who view some form of generality and coding as being possible.

For social and practical reasons, historically, AI has drawn heavily from formal logic. For example, one of the benefits of such models was that they were comparably easy to implement. Formal logic is concerned with the explicit representation of knowledge and places great emphasis on the need to codify all facts that could be of importance. This focus on knowledge as an objective truth can be traced back to e.g. the logic of Aristotle who believed that at least a particular subset of knowledge had an objective existence (Episteme) [3]. This view contrasts with that of, for example, Polanyi, who argues that no such objective truth exists and all knowledge is at some point personal and hidden (tacit) [4].

The total denial of the existence of an objective truth is problematic, since consequently there can exist no criterion to value any representation of knowledge. We 
can contrast this with the view of Kant, who regards the accordance of the cognition with its object as being presupposed in the definition of truth [5, p. 52]. Going further, he makes clear that a purely formal and universal criterion of truth cannot exist. He foregrounds the dialectic relation between the formal logic and the objects to which this logic may be applied and which are given through intuition. Such a dialectic approach overcomes the conceptual difficulties outlined above, but the consequences for computational models are not easily accounted for.

Context does not fit very well with the strict logical view on how to model the world. However, an extrememly personal and unique account of context serves little purpose in attempting generality. Context is, after all, a shared and very elusive type of knowledge. Despite the fact that humans can quite easily read context, context is hard to quantify in any formal way, and it is difficult to establish the type of knowledge that is useful in any given situation. Ekbia and Maguitman argue that this has led to context being largely ignored by the AI community [6]. Neither the relativist nor the formal logic approach to context has been very useful at producing accounts of context which resonate with the AI community, and, except for some earlier work on context and AI, Ekbia and Maguitman's observation still holds. Systemic-functional linguistics as described in the following section employs a dialectic view on context, and therefore avoids the pitfalls of the formal logic as well as the relativistic approaches.

\section{Semiotics}

Understanding meaning making and meaning making systems is the domain of Semiotics. Semiotics is commonly understood to be the study of sign systems and we here make use of systemic functional linguistics which is a social semiotic[7]. Semiotics itself has a long history and its use in computer science is not new, even if not extensive. However, it is not our intention in this paper to review the body of work surrounding semiotics though we are mindful of the impact of this work on the field today, in particular the work of Saussure [8], Peirce [9] and Voloshinov [10]. For a comprehensive account of semiotics as it is applied to computing we recommend works such as Gudwin and Queiroz [11] (in particular Bøgh Andersen and Brynskov [12] and Clarke et al. [13]) as well as de Souza [14]. The intelligent artefacts that we consider in this paper are an integral part of social interaction. They change the sense making process on the side of the human users as well as their own functioning as signs (contextualised by the users). Ideally, the artefact should be able to adapt to its use and user, and the means for this adaptation will have to be laid out by the designers.

In this research, we have used the social semiotics outlined by Halliday (see for example [15] and [16]). Halliday combines the strengths of the approaches of Saussure, Pierce, and Voloshinov. He brings together the tradition of relational thinking from Saussure, the understanding that different modalities have consequences for 
the structure of meanings from Pierce, and from Voloshinov, the insistence that the sign is social.

Halliday's Systemic Functional Theory of language (SFL) is a social semiotic theory that sets out from the assumption that humans are social beings that are inclined to interact [15]. In this paper we examine the value of the SFL notion of context, which views context as all the features of a social process relevant to meaning making. These features are organised into 3 core parameters of context: Field, Tenor and Mode, where field is "the nature of the social activity...", tenor is "the nature of social relations...", and mode is "the nature of contact..." [17]. Context, in SFL is one of four linguistic levels (see below), which are related realizationally rather than causally, meaning that patterns on one level both construe and construct patterns on another level. Halliday manages the complexity of language by modelling it as a multidimensional system. The most crucial dimensions of this multidimensional system for our purposes are: stratification and instantiation. We examine how these key notions of SFL make this model of context valuable for AI. Focusing in particular on the notion of instantiation.

Stratification: Halliday uses a stratified model of language that incorporates the levels of the expression plane (including sound systems - phonetics and phonology, gesture, pixels etc.), lexicogrammar (lexis/grammar - or wording and structure), semantics (the meaning system) and context (culture and situation - elements of the social structure as they pertain to meaning). Description on each stratum is functionally organised into systems.

Instantiation: Halliday uses a tripartite representation of language, which has language as system, language as behaviour and language as knowledge. Language as system encapsulates the abstract structure of language. This accounts for the regularised (though changeable) patternings that we see in language. It is this regularity that makes prediction and a certain degree of formalism (at least of a functional nature) possible. Language as behaviour looks at the activity of language, while language as knowledge looks at the way in which we know language. But we do not do these things independently. We do not know language as a set of abstract rules. Rather we know language in the sense of knowing how to use it, in the sense of knowing how to communicate with others [15]. In practice these things occur together. When we try to build a device, it is language behaviour and knowledge that we face, yet it is the seemingly inaccessible system that we need to encode in order to produce intelligent seeming behaviours and knowledge in the device.

The concept that encapsulates this problem is what Halliday calls the cline of instantiation. This is a way of looking at the relationship between System (which at the level of context means the culture) and Instance (which at the level of context means the situation that we are in). This is represented in figure 1 . Here we see in the foreground the system view of language, and its grounding in the instance.

The formalization of a level of context as part of a polysystemic representation of language has long been emphasized in the work of systemic functional linguists, especially Halliday and Hasan [18]. It is the dialectic approach of systemic functional linguistics which avoids the problem of vastness and that of uniqueness. 


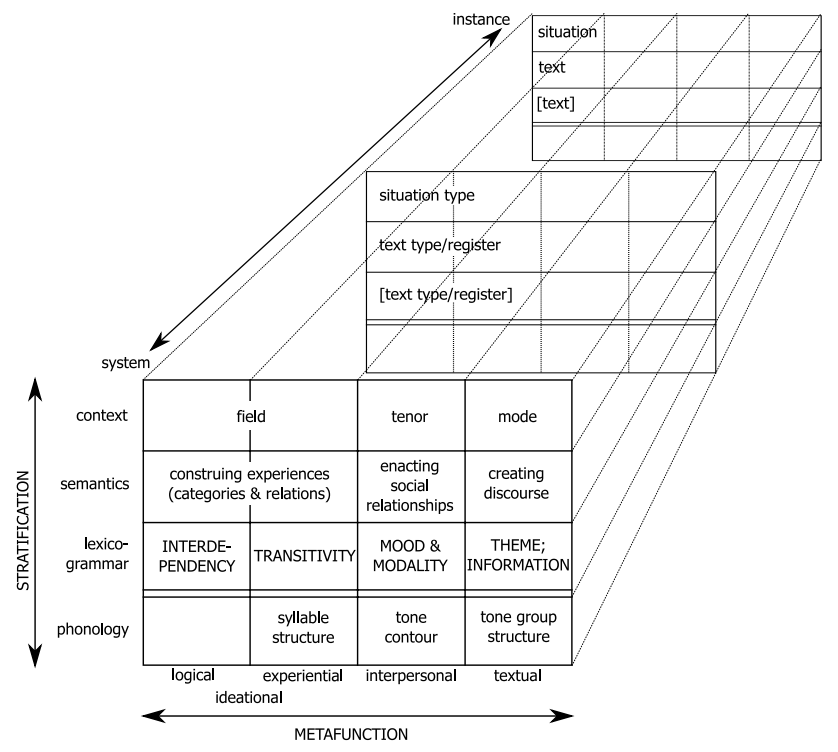

Fig. 1 The dimensions of language - Halliday and Matthiessen

Instances that share a similar function, e.g. instances of ward rounds in hospitals, typically share a similar structure. Halliday refers to these situation types as registers and they represent a functional variety of language [16]. The value of register is that we do not have to describe everything. Register can be thought of as an aperture on the culture. So, we are not faced with the full complexity of the culture. This does not mean that we do not keep the culture in mind. Any picture of a part of the system necessarily has the full system behind it. With register we set out from the instance, but keep in mind that each instance is a take on the system. Our notion of what constitutes an instance is shaped by our understanding of the culture/system. So, although Halliday represents the relationship between system and instance as a cline of instantiation, it is probably best understood as a dialectic since the two are never actually possible without each other. Register does not so much sit between system and instance, as it is a take on system and instance at the one time. It is the culture brought to bear on the instance of the social process.

For ambient intelligence, this means that we are not faced with the unhelpful uniqueness of each instance, because we are viewing it through the system and therefore foregrounding the shared aspects. Neither are we confronted with the seemingly impossible task of transcribing the infinity of culture, because we are viewing the culture through the aperture of the instance. 


\section{Semiotics in Ambient Intelligence}

In this section, we will give our basic understanding of how semiotics can be used to understand the peculiarities of user interaction with ambient intelligent systems. The basic concept of the chosen interpretion of semiotics is the sign, a triadic relation of a signifier, a signified, and object. We look at the process of sense-making, where a representation (signifier) and its mental image (signified) refer to an entity (object) (the meaning of a sign is not contained within a symbol, it needs its interpretation).

On the background of semiotics, meaningful human communication is a sign process. It is a process of exchanging and interpreting symbols referring to objects. The user of a computer systems sees his interaction with this system on this background. When typing a letter, he does not send mere symbols, but signs to the computer, and the feedback from the machine, the pixels on the screen, are interpreted as signs: to the user, the computer is a "semiotic machine". The question that arises is whether a computer is actually itself taking part in the sense making process.

On one hand, following for example Kant, human understanding has as a necessary constituent the ability to conceptualise perceived phenomena through an active, discursive process of making sense of the intuitive perception [5, p. 58]. Following this understanding, computer systems are only processing signals, lacking the necessary interpreting capabilities humans have. They only manipulate symbols without conceptualising them.

On the other hand, we can take a pragmaticist approach, following for example Peirce and Dewey, and focus not on whether the machine is itself a sense maker, but on how its use changes the ongoing socio-technical process, and whether it can mediate the sense making process. From this point of view, the computer can be a sense making agent if its actions are appropriate in terms of the user's expectations.

Both approaches lead to a change in the issues we deal with when constructing an ambient intelligent system. The problem is transformed from one where the issue is to build a machine which itself realises a sense making process to one in which the issue is to build a computer thats actions are appropriate for the situation it is in and which exhibits sufficient sign processing behaviour.

We argue that, in order to make a pervasive, ambient intelligent system that behaves intelligently in a situation, it must be able to execute actions that make a difference to the overall sense making process in a given context. This differs from the interaction with traditional systems in which case the sense-making falls wholly on the side of the human user: You do not expect a text processor to understand your letter, but you expect an ambient intelligent system to display behaviour suggesting that it understands relevant parts of the situation you are in. When interacting with ambient intelligent systems, the user should be facilitated to subscribe to the sense making abilities of the artefacts. We consider the ability of the system to deal with concepts which have no direct material reference to be important to achieve this goal. 


\section{Abstract Concepts}

Abstraction, or the ability to create a more general category from a set of specifics by whatever principle, is arguably one of the most useful mental tools that humans possess [19]. Indeed [20] suggests that the abstract categories that form part of our everyday life and language, are typically below conscious attention and only become apparent through linguistic analysis.

Such abstraction, though important to human intelligence, presents a challenge for modelling in ambient intelligence. Consider the meanings of the word 'Emergency'. Emergency has numerous meanings depending on the context in which it occurs. For the purposes of our discussion we will here limit ourselves to the hospital environment. In the hospital environment, 'emergency' has specific meanings that are distinct from the meanings in other contexts. Not only are there hospital specific meanings (culture specific), but the meaning varies according to the situation as well (situation specific). Within the hospital domain the term emergency may be understood to have two distinct meanings. Firstly, the term may mean the emergency department of the hospital. This is a concrete concept with a direct material referent of a place: the emergency department of the hospital. Drawing on the notion of stratification, we can see that this concept is typically realized in the lexicogrammar ${ }^{1}$ by use of the specific deictic (e.g. 'the emergency department'), and by the possibility of using it as a circumstance location spatial (e.g. 'in the emergency department').

Secondly, the term may mean an emergency. This meaning of the term is an abstract concept with no direct referent in the material setting, referring instead to a state. This term is realized in the lexicogrammar by use of a non-specific deictic (e.g. 'an emergency') and may, if used in the past tense, use the specific deictic accompanied by a circumstance of location either spatial or temporal (e.g. 'the emergency in F ward' or 'the emergency this morning'). Note that here it is not the emergency that is the circumstance, but either time or location.

Our focus in this paper is on the second of these meanings. This meaning, an emergency, may be understood to refer to a complex set of actions and relations that constitute an interruption to the normal flow of a social process. This interruption may be:

- Culture based: deriving from the function of the broader hospital culture, or,

- Context based: deriving from variation within the structure of the social process itself.

It is this relation between culture based and context based meanings that is explored below.

To function intelligently in context, artefacts must be able to recognise 'emergency' and respond appropriately. They may need, for example, to "be quiet" while the doctor deals with an 'emergency' or they may need to "provide new information" needed by the doctor in an 'emergency'. To account for these complexities,

\footnotetext{
${ }^{1}$ This makes use of the relationship between patterns on different levels of language. For details, see section 3
} 
a rich, but targeted, description of the culture is needed. To do this we will use the notions of register and generic structure potential [21] and a contextual model of language.

In order to establish what emergency means in this context we need to see its place in the system. That means we need to understand how it fits within the hospital culture. Understanding the richness of the culture is part of adequately embedding a device into that culture. Not doing so runs the risk of producing an artefact unsuited to its purpose and thus unintelligent. Part of what makes something (appear) intelligent is the ability to read and respond to the context. Context here is not just the immediate setting of the artefacts, (the context of situation), but the culture of which that setting is a part. Ward rounds then must be seen from the perspective of how they fit into the hospital culture. Within the function of the hospital, which is the restoration of health, the function of ward rounds is to monitor health. Because it has a 'monitoring' function within the hospital culture, it will be possible for the ward round to be interrupted by 'emergencies' from the wider hospital, since the function of the hospital overrides that of the ward round in terms of urgency.

By understanding the function of the ward round, and its contextual configuration, it is possible to state a generic structure potential for a ward round. A generic structure potential is a statement of the likely structure of a context. A generic structure however does not mean that there will not be variation. The notion of a ward round for example, is itself a functional abstraction ${ }^{2}$ of all the behaviours, relations, and communications that go into completing a ward round. We are able to recognise from experience that certain behaviours by different participants, combined with certain roles and relations (e.g. ward doctor, ward nurse, patient, specialist) combined with the exchange of certain types of information (receiving information, requesting information, giving information) together constitute a ward round. None of these behaviours, relations or communications on their own constitutes a ward round, the ward round is identified by all of these things together.

Understanding the function both of the hospital within society and the ward round within that environment, facilitates the construction of a picture of the generic structure of a ward round and its place within the broader hospital culture. This enables a better understanding of the likely meaning of abstract concepts such as 'emergency'. Based on these conceptions of the ward round context, it is possible to posit the existence of two broad categories of emergency: those constituting an interruption to the ward round (when the hospital culture impinges on the ward round) and those constituting a change to the ward round (when there is internal variation in the ward round context). Because the first involves changes to the field (a new topic, ward, and focus), tenor (very different participants and role relations), it is likely to require a "new information response". This is because the field, tenor and mode settings have changed so much that it is now a new context and will thus require different information to suit this new context. The second will not involve changes to the mode or tenor, and only minor changes to the field. Thus it is likely to require a "be quiet and await query" response. This is because this is not a new context, it

\footnotetext{
${ }^{2}$ Here used to refer to the means by which abstraction is made, i.e. by considering the function of the behaviour.
} 
is simply variation within the structure of the ward round. By utilising the notion of register to limit what we have to consider in the culture, and the concept of generic structure potential to model a typical view of the situation based on our study of the instances, we are able to better understand the context of the ward round and how to model abstract concepts for this context.

\section{Conclusion and further work}

In this paper we have considerered one of several ways that semiotics can be made fruitful in ambient intelligence. This research has suggested many areas of future investigation. In this project we have focused on the individual, but the sign making process is a negotiated process. It is not simply one meaner that has to be considered. In any exchange there are always at least two meaners, and more typically more than two. Multiparticipant communication represents a challenge to modelling. We have to keep in mind that others may share our conceptualisations and meanings only to a certain extent. When ambient intelligent systems link different people this is an important thing to remember. The closer a person is in our social network the more likely they are to share our meanings, while the further out in our social network the less likely they are to share meanings. In the hospital environment, ambient intelligent devices can belong to different groups of users. Should we model them in a way that the assistant of a nurse is more likely to share concepts with the assistant of another nurse than that of a physician?

Ambient intelligent systems will have deal with these kinds of challenges. Another point to consider is where in the network the system itself sits. What is the relation of the system to its user? To other pervasive devices? To their users? We are effectively dealing with a case of dialectal variation. Certain users may find some signs transparent and others not, while other users may find the exact opposite. If ambient intelligent systems are used to link people how do they best utilise signs to do this? This issue becomes very important when health care professionals from different cultural and language backgrounds have to interact.

Another issue we would like to explore further is the extent to which it is possible to relate a semiotic approach to ambient intelligent systems design to other sociotechnical theories already in use in the field of ambient intelligence. A promising candidate is for example activity theory. Bødker and Andersen have outlined some properties of a socio-technical approach taking advantage of ideas from both theoretical frameworks [22], and we would like to extend this to cover specific aspects of SFL and Cultural-Historical Activity Theory (CHAT). This will potentially extend the number of projects from which we can borrow findings, meaning a richer description of the hospital environment.

Another point we have not fully explored yet is the relation of concepts from SFL with specific methods from the field of artificial intelligence. For example, the notion of genres in SFL seems to be a likely candidate for knowledge poor lazy learning mechanisms, while the descriptive power of register might be exploitable 
in knowledge intensive or ontology based approaches. A promising candidate to combine these aspects is knowledge-intensive case-based reasoning.

\section{References}

1. Leake, D.B.: Goal-based explanation evaluation. In: Goal-Driven Learning. MIT Press, Cambridge (1995) 251-285

2. Ducatel, K., Bogdanowicz, M., Scapolo, F., Leijten, J., Burgelman, J.C.: ISTAG scenarios for ambient intelligence in 2010. Technical report, IST Advisory Group (2001)

3. Aristotle: Analytica Posteriora. Akademie (1998)

4. Polanyi, M.: Personal Knowledge: Towards a Post-critical Philosophy. N.Y.: Harper \& Row (1964)

5. Kant, I.: Kritik der reinen Vernunft (2. Auflage). Akademie (1787)

6. Ekbia, H.R., Maguitman, A.G.: Context and relevance: A pragmatic approach. Lecture Notes in Computer Science 2116 (2001) 156-169

7. Hodge, R., Kress, G.: Social Semiotics. Cornell, UK (1988)

8. Saussure, F.d.: Course in General Linguistics. McGraw-Hill (1966)

9. Peirce, C.S.: New elements (kaina stoicheia). In Eisele, C., ed.: The New Elements of Mathematics by Charles S. Peirce. Volume 4, Mathematical Philosophy. (1904) 235-263

10. Voloshinov, V.N.: Marxism and the Philosophy of language. Seminar Press, New York (1973)

11. Gudwin, R., Queiroz, J.: Semiotics and Intelligent Systems Development. IGI Publishing, Hershey, PA, USA (2006)

12. Andersen, P.B., Brynskov, M.: The semiotics of smart appliances and pervasive computing. [11] 211-255

13. Clarke, R., Ghose, A., Krishna, A.: Systemic semiotics as a basis for an agent oriented conceptual modeling methodology. [11] 256-285

14. de Souza, C.S.: The Semiotic Engineering of Human-Computer Interaction (Acting with Technology). The MIT Press (2005) QA76.9.H85 .D465 2005.

15. Halliday, M.A.: Language as a Social Semiotic: the social interpretation of language and meaning. University Park Press (1978)

16. Halliday, M.A., Matthiessen, C.M.: An Introduction to Functional Grammar, Third edition. Arnold, London, UK (2004)

17. Hasan, R.: Speaking with reference to context. In Ghadessy, M., ed.: Text and Context in Functional Linguistics. John Benjamins, Amsterdam (1999)

18. Halliday, M.A., Hasan, R.: Language, Context, and Text: aspects of language in a scoialsemiotic perspective. Deakin University Pres, Geelong, Australia (1985)

19. Butt, D.: Multimodal representations of remote past and projected futures: from human origins to genetic counselling. In Amano, M.C., ed.: Multimodality: towards the most efficient communications by humans. $21^{\text {st }}$ century COE Program International Conference Series NO. 6, Graduate school of letters, Nagoya University (2006)

20. Whorf, B.L.: Language, Thought and Reality (ed. J. Carroll). MIT Press, Cambridge, MA (1956)

21. Hasan, R.: Situation and the definition of genre. In Grimshaw, A., ed.: What's going on here? Complementary Analysis of Professional Talk: volume 2 of the multiple analysis project. Ablex, Norwood N.J. (1994)

22. B $ø$ dker, S., Andersen, P.B.: Complex mediation. Journal of Human Computer Interaction 20 (2005) 353-402 